\title{
Association of Hemorheology With High-Sensitivity Cardiac Troponin T Levels in Patients With Type 2 Diabetes Mellitus Assessed by Microchannel Array Flow Analyzer
}

\author{
Takashi Hitsumoto
}

\begin{abstract}
Background: Recent studies on high-sensitivity cardiac troponin $\mathrm{T}$ (hs-cTnT) and whole blood passage time (WBPT), estimated by hemorheology assessed with the microchannel array flow analyzer (MC-FAN), have revealed the significance of cardiovascular risk factors. However, there are no known reports on the relationship between these two clinical parameters. This cross-sectional study aimed to clarify the associations between WBPT and hs-cTnT levels in patients with type 2 diabetes mellitus from the perspective of subclinical myocardial injury or the primary prevention of cardiovascular events.

Methods: A total of 301 outpatients (men, 116; women, 185; mean age, $65 \pm 11$ years), with no history of cardiovascular diseases, undergoing treatment for diabetes mellitus were enrolled. Hs-cTnT levels and WBPT were measured using a commercial device, and the relationship between hs-cTnT levels and various clinical parameters, including WBPT, was examined.
\end{abstract}

Results: Hs-cTnT levels were detected in 261 (86.7\%) patients. WBPT was significantly higher in patients with detectable hs-cTnT levels than in those with undetectable hs-cTnT levels $(64 \pm 18 \mathrm{~s}$ vs. $51 \pm 13 \mathrm{~s}, \mathrm{P}<0.001)$. In patients with detectable hs-cTnT levels, there was a significantly positive correlation between WBPT and hs-cTnT levels $(r=0.40 ; \mathrm{P}<0.001)$. Furthermore, multiple regression analysis revealed that WBPT $(\beta=0.24 ; \mathrm{P}<0.001)$ was an independent variable when hs-cTnT was a subordinate factor. Conversely, patients with high ( $\geq 70 \mathrm{~s}$; odds ratio, $5.3(95 \% \mathrm{CI}, 1.6-16.1) ; \mathrm{P}<0.01)$ and median (50.2 - $69.8 \mathrm{~s}$; odds ratio, 3.8 (95\% CI, 1.2 - 12.4); $\mathrm{P}<0.05)$ WBPT had a significantly higher risk for high hs-cTnT levels $(\geq 0.014$ $\mathrm{ng} / \mathrm{mL})$ than those with low WBPT $(\leq 50.0 \mathrm{~s})$.

Conclusions: The results of this study indicated independent associations of hemorheology with hs-cTnT in patients with type 2 diabetes mellitus assessed by MC-FAN. We suggest that an increase in hscTnT levels can be prevented by maintaining WBPT at $\leq 50.0 \mathrm{~s}$.

Keywords: Hemorheology; Microchannel methods; High-sensitivity

Manuscript submitted November 11, 2017, accepted November 23, 2017

Hitsumoto Medical Clinic, 2-7-7, Takezakicyou, Shimonoseki-City, Yamaguchi 750-0025, Japan. Email: thitsu@jcom.home.ne.jp

doi: https://doi.org/10.14740/cr632w troponin T; Advanced glycation end-products; Oxidative stress; Cardio-ankle vascular index; Type 2 diabetes mellitus

\section{Introduction}

Diabetes mellitus is known to cause a progression in myocardial injury in the subclinical stage of heart failure, and several mechanisms, such as hyperglycemia-mediated coronary microvascular dysfunction and oxidative stress, are thought to cause myocardial injury [1-3]. Conversely, blood concentration of high-sensitivity cardiac troponin $\mathrm{T}$ (hs-cTnT) is known to be a useful biomarker to evaluate myocardial injury in the clinical stage [4]. Epidemiological studies have reported hscTnT as a predictor of cardiovascular events not only in the general population but also in patients with type 2 diabetes mellitus [5-7].

The impairment of hemorheology is considered an important factor in the incidence of cardiovascular events and atherosclerosis $[8,9]$. In recent years, the microchannel array flow analyzer (MC-FAN), which is a commercial device that assesses hemorheology using microscopic images, has been introduced in clinical settings [10]. MC-FAN has a simple methodology and is superior to other methods on the basis of the accuracy of channel dimensions and high reproducibility. Furthermore, some clinical studies have reported a significant relationship between increased whole blood passage time (WBPT), which is measured using MC-FAN, and cardiovascular risk factors or diseases [11-14].

To the best of our knowledge, there are no reports on the relationship between WBPT and hs-cTnT levels in patients with type 2 diabetes mellitus; therefore, this study aimed to elucidate the associations of WBPT and hs-cTnT in patients with type 2 diabetes mellitus from the perspective of subclinical myocardial injury or the primary prevention of cardiovascular events.

\section{Materials and Methods}

\section{Study population}

This study was conducted at the Hitsumoto Medical Clinic 


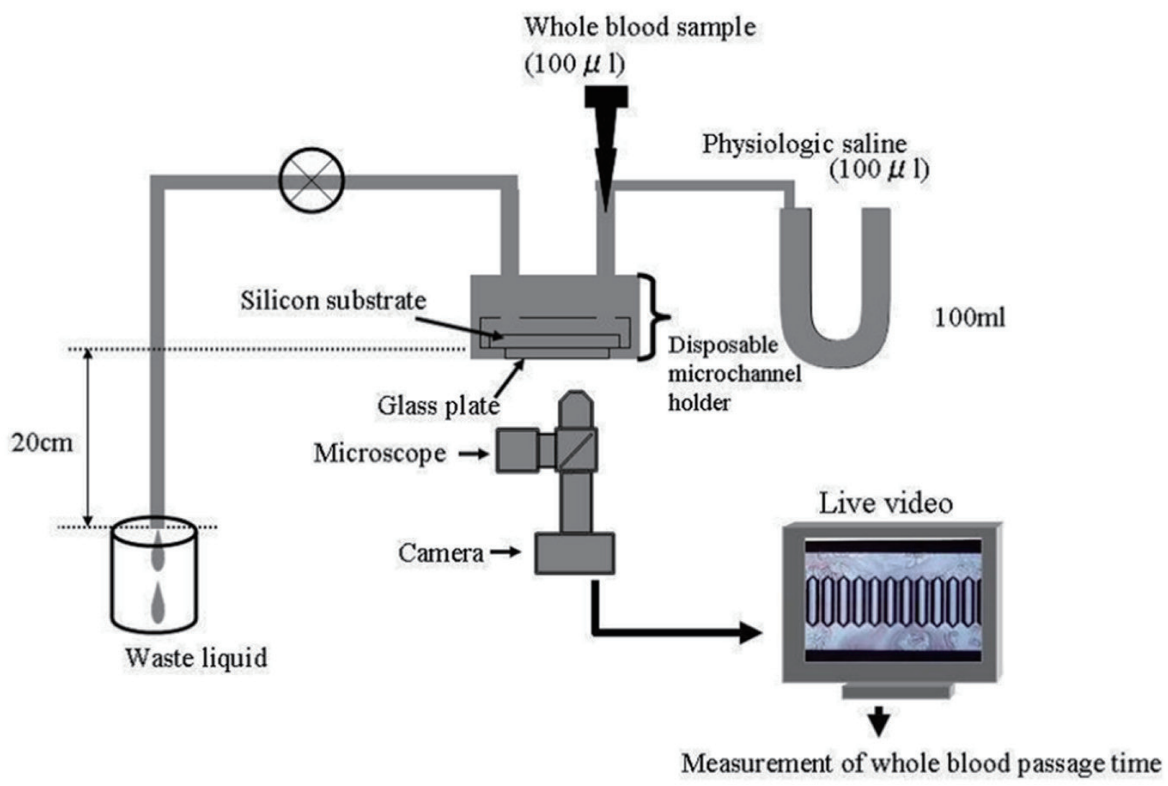

Figure 1. System of microchannel array flow analyzer. The microchannel passage time of $100 \mu \mathrm{L}$ of physiologic saline was measured as a control, and then that of venous whole blood obtained from the subjects with $5 \%$ heparinization was determined. The whole blood passage time of the subjects was expressed after correction for the passage time of physiologic saline. Inter- and intra-assay coefficients of variation for whole blood passage time were $8 \%$ and $5 \%$, respectively.

in Shimonoseki City, Japan, between April 2015 and March 2017. The study population comprised 301 outpatients (males, $116(38.5 \%)$ and females, 185 (61.5\%); mean age, $65 \pm 11$ years) undergoing treatment for type 2 diabetes mellitus. No patient had a history of cardiovascular events, such as coronary artery, cerebrovascular, and perivascular diseases or heart failure. All patients provided informed consent, and the study protocol was approved by the Local Ethics Committee of the Hitsumoto Medical Clinic.

\section{Evaluation of hemorheology by MC-FAN}

Hemorheology was evaluated by measuring WBPT using a MC-FAN HR300 rheometer as the commercial device (MC Healthcare Inc., Tokyo, Japan) (Fig. 1) [10, 12]. Briefly, the microchannel passage time for $100 \mu \mathrm{L}$ of physiological saline as a control was measured, followed by that for $100 \mu \mathrm{L}$ of heparinized whole blood samples obtained from patients. WBPT for patients was corrected for the passage time of physiological saline. The microchannel formation was $7 \mu \mathrm{m}$ wide, $30 \mu \mathrm{m}$ long and $4.5 \mu \mathrm{m}$ deep. WBPT was measured within $60 \mathrm{~min}$ of blood sampling. The inter- and intra-assay coefficients of variation for WBPT were $8 \%$ and 5\%, respectively.

\section{Estimation of clinical parameters}

Various clinical parameters, classic coronary risk factors, glucose-related parameters, blood cell count, kidney function, left ventricular hypertrophy estimated by electrocardiography, brain natriuretic peptide (BNP), oxidative stress, arterial func- tion, and hs-cTnT levels were evaluated. The degree of obesity was measured using body mass index, calculated as weight in kilograms divided by height in meters squared. Current smoking was defined as smoking at least one cigarette per day during the previous 28 days. Hypertension was defined as systolic blood pressure of $\geq 140 \mathrm{~mm} \mathrm{Hg}$, diastolic blood pressure of $\geq 90 \mathrm{~mm} \mathrm{Hg}$, or the use of antihypertensive treatment. Dyslipidemia was defined as low-density lipoprotein cholesterol levels of $\geq 140 \mathrm{mg} / \mathrm{dL}$, HDL cholesterol levels of $\leq 40 \mathrm{mg} / \mathrm{dL}$, triglycerides levels of $\geq 150 \mathrm{mg} / \mathrm{dL}$, or the use of antidyslipidemic treatment. Skin autofluorescence (AF) as a marker of advanced glycation end products (AGEs) in tissues was measured using a commercial instrument (AGE ReaderTM, DiagnOptics, Groningen, The Netherlands), as described previously [15]. Briefly, AF was defined as the average light intensity per nanometer in the range between 300 and $420 \mathrm{~nm}$. AF levels were expressed in arbitrary units. All patients were measured in a seated position at the volar side of the lower arm, approximately $10-15 \mathrm{~cm}$ below the elbow fold. The severity of left ventricular hypertrophy was evaluated using Cornell ( $\mathrm{R}$ wave in $\mathrm{aVL}+\mathrm{S}$ wave in V3) electrocardiographic voltage calculations [16]. Arterial function was evaluated by cardio-ankle vascular index (CAVI) using a VaSera CAVI instrument (Fukuda Denshi Inc., Tokyo, Japan) according to the methods described previously [17]. Briefly, brachial and ankle pulse waves were determined using inflatable cuffs by maintaining the pressure between 30 and $50 \mathrm{~mm} \mathrm{Hg}$ to ensure a minimal effect of the cuffs' pressure on systemic hemodynamics. Blood and pulse pressures were simultaneously measured with patients in the supine position. CAVI was measured after the patient rested for $10 \mathrm{~min}$ in a quiet room. The average coefficient of variation of CAVI has been shown to be $<5 \%$, which is small enough for 
Table 1. Patient Characteristics

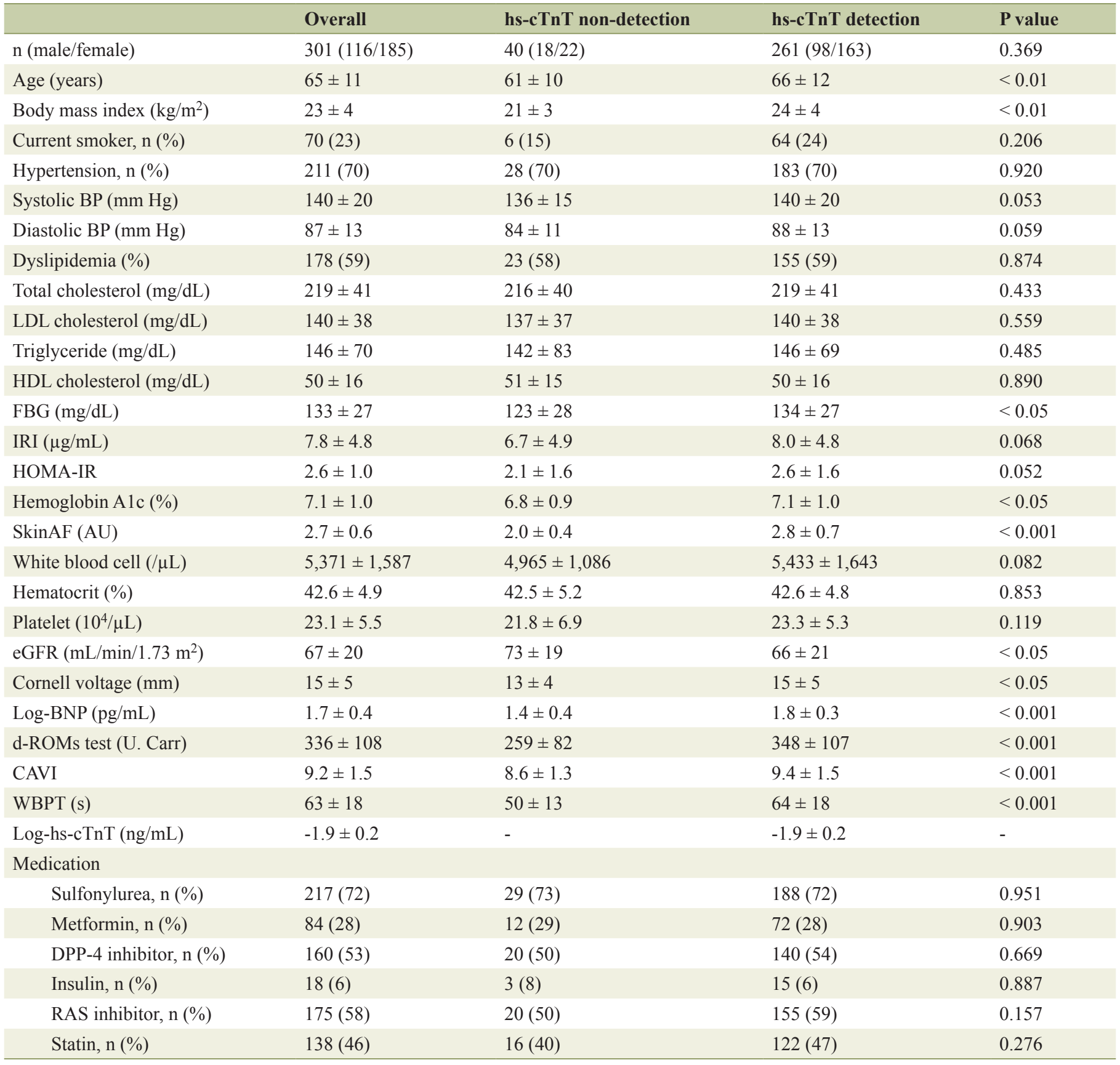

Data are expressed as mean \pm standard deviation. hs-cTnT: high-sensitivity cardiac troponin T; BP: blood pressure; LDL: low-density lipoprotein; HDL: high-density lipoprotein; FBG: fasting blood glucose; IRI: immunoreactive insulin; HOMA-IR: homeostatic model assessment of insulin resistance; AF: autofluorescence; eGFR: estimated glomerular filtration rate; BNP: brain natriuretic peptide; d-ROMs: derivatives of reactive oxygen metabolites; CAVI: cardio-ankle vascular index; WBPT: whole blood passage time; DPP: dipeptidyl peptidase; RAS: renin-angiotensin system.

clinical use and indicates good reproducibility.

\section{Evaluation of blood parameters}

Blood samples were collected from the antecubital vein in the morning after $12 \mathrm{~h}$ of fasting. Total cholesterol and triglyceride concentrations were measured using standard enzymatic methods. Serum high-density lipoprotein cholesterol concentrations were measured by selective inhibition, and serum low-density lipoprotein cholesterol concentrations were measured using the Friedewald equation [18]. Patients with a serum triglyceride concentration of $\geq 400 \mathrm{mg} / \mathrm{dL}$ were excluded, considering the accuracy of this method. Glucose and insulin con- 
centrations were measured using the glucose oxidase method and enzyme immunoassay, respectively. To measure insulin resistance, HOMA-IR was calculated as follows [19]: HOMA$\mathrm{IR}=$ fasting glucose concentration $(\mathrm{mg} / \mathrm{dL}) \times$ fasting insulin concentration $(\mu \mathrm{g} / \mathrm{mL}) / 405$. Hemoglobin A1c levels were expressed according to the National Glycohemoglobin Standardization Program standards. Estimated glomerular filtration rate (eGFR) was calculated using the adjusted Modification of Diet in Renal Disease Study equation, which was proposed by the working group of the Japanese Chronic Kidney Disease Initiative [20]. The blood concentration of BNP was measured using a commercial kit (SHIONOSPOT Reader; Shionogi \& Co., Ltd, Osaka, Japan). Reactive oxygen metabolites (dROMs) test as a marker of oxidative stress was conducted using a commercial kit (Diacron; Grosseto, Italy) [21]. Hs-cTnT levels were also measured using a commercial kit (Roche Diagnostics, Basel, Switzerland) [22]. In the hs-cTnT assay, the lower limit of detection was $0.003 \mathrm{ng} / \mathrm{mL}$.

\section{Statistical analysis}

A commercially available statistical software program (Stat View-J 5.0; HULINKS Inc., Tokyo, Japan) was used for all statistical analyses. Data are expressed as mean \pm SD. Betweengroup comparisons were performed using Student's $t$-test or Mann-Whitney's U test, and the correlation coefficient was estimated using the Spearman rank-order correlation analysis. Multivariate analysis was conducted using multiple regression or multiple logistic regression analysis. P value of $<0.05$ was considered statistically significant.

\section{Results}

Table 1 presents patients' characteristics. Hs-cTnT levels were detected in $261(86.7 \%)$ patients. Age, body mass index, fasting blood glucose levels, hemoglobin A1c, skin AF, Cornell voltage, BNP, d-ROMs test, and CAVI were significantly higher, and eGFR was significantly lower in patients with detectable hs-cTnT levels. WBPT was significantly higher in patients with detectable hs-cTnT levels.

Correlations among hs-cTnT, WBPT, and various clinical parameters in the hs-cTnT detection cohort are presented in Table 2. Age, body mass index, presence of hypertension, hemoglobin A1c, skin AF, eGFR, Cornell voltage, BNP, d-ROMs test, and CAVI were significantly correlated with hs-cTnT. Conversely, body mass index, current smoking, presence of hypertension, serum triglyceride concentration, hemoglobin A1c, skin AF, white blood cell count, hematocrit, eGFR, d-ROMs test, and CAVI were significantly correlated with WBPT. Correlations between hs-cTnT and WBPT are shown in Figure 2. Significantly positive correlations were observed in the two parameters.

Table 3 shows the results of multiple regression analysis for hs-cTnT or WBPT as a subordinate factor. Explanatory factors were selected by either checking multicollinearity among variables or by the stepwise method. CAVI, WBPT, BNP, skin $\mathrm{AF}, \mathrm{d}-\mathrm{ROMs}$ test, and age were selected as independent vari-
Table 2. Correlation Between Hs-cTnT, WBPT and Clinical Parameters in Hs-cTnT Detectable Patients

\begin{tabular}{|c|c|c|}
\hline & r & \\
\hline & Log-hs-cTnT & WBPT \\
\hline Sex $($ female $=0$, male $=1)$ & 0.04 & 0.06 \\
\hline Age & $0.19 * *$ & 0.03 \\
\hline Body mass index & $0.14 *$ & $0.18^{* *}$ \\
\hline Current smoker $($ no $=0$, yes $=1)$ & 0.07 & $0.20 * *$ \\
\hline Hypertension $($ no $=0$, yes $=1)$ & $0.12 *$ & $0.13 *$ \\
\hline Systolic BP & 0.08 & 0.10 \\
\hline Diastolic BP & 0.06 & 0.08 \\
\hline Dyslipidemia $($ no $=0$, yes $=1)$ & -0.03 & -0.01 \\
\hline Total cholesterol & -0.04 & 0.08 \\
\hline LDL cholesterol & -0.05 & 0.07 \\
\hline Triglyceride & -0.03 & $0.21 * *$ \\
\hline HDL cholesterol & -0.07 & -0.09 \\
\hline FBG & 0.07 & 0.11 \\
\hline IRI & 0.05 & 0.07 \\
\hline HOMA-IR & 0.06 & 0.10 \\
\hline Hemoglobin A1c & $0.13 *$ & $0.13 *$ \\
\hline Skin AF & $0.32 * * *$ & $0.36 * * *$ \\
\hline White blood cell & 0.06 & $0.13 *$ \\
\hline Hematocrit & 0.05 & $0.20 * *$ \\
\hline Platelet & 0.03 & 0.08 \\
\hline eGFR & $-0.19 * *$ & $-0.14 *$ \\
\hline Cornell voltage & $0.22 * * *$ & 0.10 \\
\hline Log-BNP & $0.35^{* * *}$ & 0.07 \\
\hline d-ROMs test & $0.30 * * *$ & $0.40 * * *$ \\
\hline CAVI & $0.41 * * *$ & $0.42 * * *$ \\
\hline Sulfonylurea $($ no $=0$, yes $=1)$ & -0.05 & -0.08 \\
\hline Metformin $($ no $=0$, yes $=1)$ & -0.03 & -0.03 \\
\hline DPP-4 inhibitor $($ no $=0$, yes $=1)$ & -0.05 & -0.06 \\
\hline Insulin $($ no $=0$, yes $=1)$ & -0.05 & -0.06 \\
\hline RAS inhibitor $($ no $=0$, yes $=1)$ & -0.09 & -0.07 \\
\hline Statin $($ no $=0$, yes $=1)$ & -0.07 & -0.09 \\
\hline
\end{tabular}

r expressed correlation coefficient. ${ }^{*} \mathrm{P}<0.05$, ${ }^{* *} \mathrm{P}<0.01$, ${ }^{* * *} \mathrm{P}<0.001$. hs-cTnT: high-sensitivity cardiac troponin T; BP: blood pressure; LDL: low-density lipoprotein; HDL: high-density lipoprotein; FBG: fasting blood glucose; IRI: immunoreactive insulin; HOMA-IR: homeostatic model assessment of insulin resistance; AF: autofluorescence; eGFR: estimated glomerular filtration rate; BNP: brain natriuretic peptide; $d$ ROMs: derivatives of reactive oxygen metabolites; CAVI: cardio-ankle vascular index; WBPT: whole blood passage time; DPP: dipeptidyl peptidase; RAS: renin-angiotensin system.

ables when hs-cTnT was used as a subordinate factor, whereas skin AF, hs-cTnT, d-ROMs test, CAVI, hematocrit, and smoking habits were selected as independent variables when WBPT was a subordinate factor. 


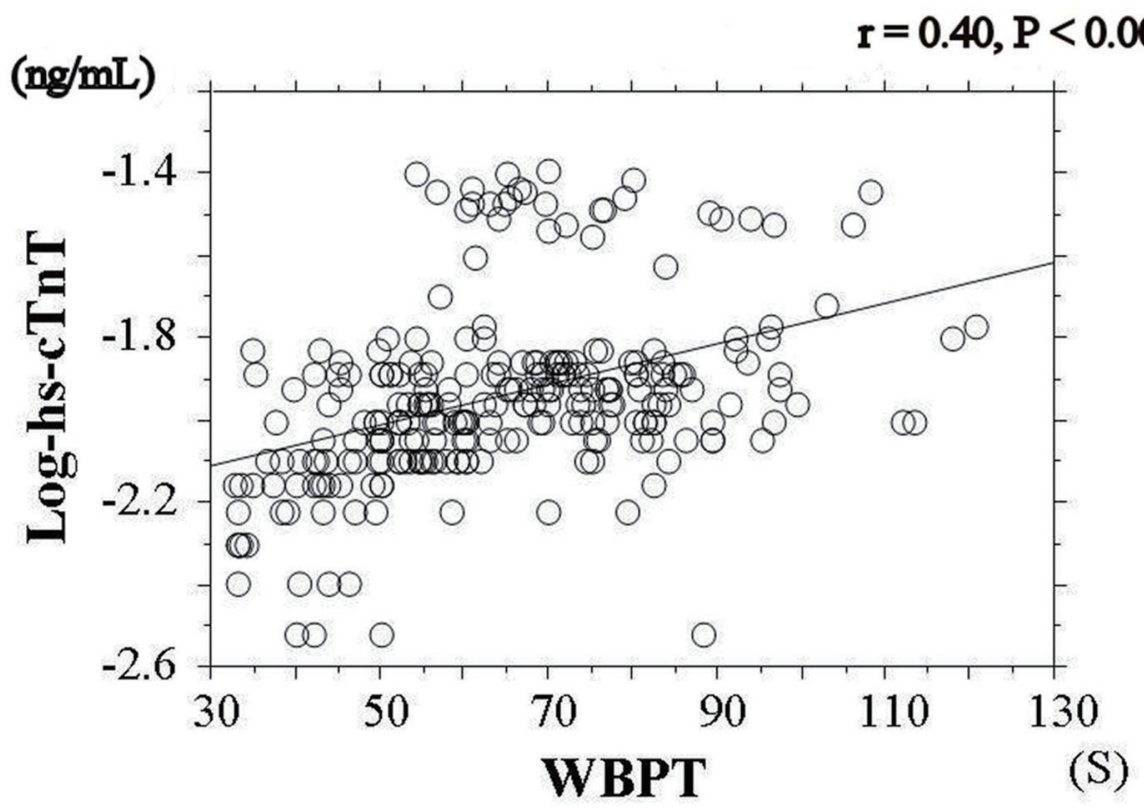

Figure 2. Relationship between hs-cTnT and WBPT. There is significantly positive correlations between log-hs-cTnT and WBPT $(r=0.40, P<0.001)$. hs-cTnT: high sensitivity cardiac troponin T; WBPT: whole blood passage time.

To illustrate the simple threshold of WBPT for detecting high hs-cTnT levels (hs-cTnT $\geq 0.014 \mathrm{ng} / \mathrm{mL}$ ), patients were divided into three groups based on WBPT, and multiple logis-

Table 3. Multiple Regression Analysis

\begin{tabular}{|lll|}
\hline Explanatory factor & $\boldsymbol{\beta}$ & P value \\
\hline (A) & & \\
CAVI & 0.27 & $<0.001$ \\
WBPT & 0.24 & $<0.001$ \\
Log-BNP & 0.21 & $<0.01$ \\
Skin AF & 0.20 & $<0.01$ \\
d-ROMs test & 0.19 & $<0.05$ \\
Age & 0.18 & $<0.05$ \\
Cornell voltage & 0.09 & 0.104 \\
eGFR & -0.06 & 0.251 \\
(B) & & $<0.001$ \\
Skin AF & 0.36 & $<0.001$ \\
Log-hs-cTnT & 0.29 & $<0.01$ \\
d-ROMs test & 0.19 & $<0.05$ \\
CAVI & 0.15 & $<0.05$ \\
Hematocrit & 0.13 & $<0.05$ \\
Current smoker & 0.12 & 0.387 \\
Triglyceride & 0.04 & \\
\hline
\end{tabular}

(A) Subordinate factor is $h s-c T n T R^{2}=0.3$. (B) Subordinate factor is WBPT $\mathrm{R}^{2}=0.44$. $\mathrm{CAVI}$ : cardio-ankle vascular index; WBPT: whole blood passage time; BNP: brain natriuretic peptide; AF: autofluorescence; $d$ ROMs: derivatives of reactive oxygen metabolite; eGFR: estimated glomerular filtration rate; hs-cTnT: high-sensitivity cardiac troponin T. tic regression analysis was performed (Fig. 3). Patients with a high ( $\geq 70 \mathrm{~s}$; odds ratio, $5.3 ; 95 \% \mathrm{CI}, 1.6-16.1 ; \mathrm{P}<0.01)$ and median (50.2 - $69.8 \mathrm{~s}$; odds ratio, 3.8; 95\% CI: 1.2 - 12.4; $\mathrm{P}<$ $0.05)$ WBPT had a significantly higher risk of high hs-cTnT levels than those with a low WBPT $(\leq 50.0 \mathrm{~s})$.

\section{Discussion}

Studies using MC-FAN indicated that mean WBPT values in

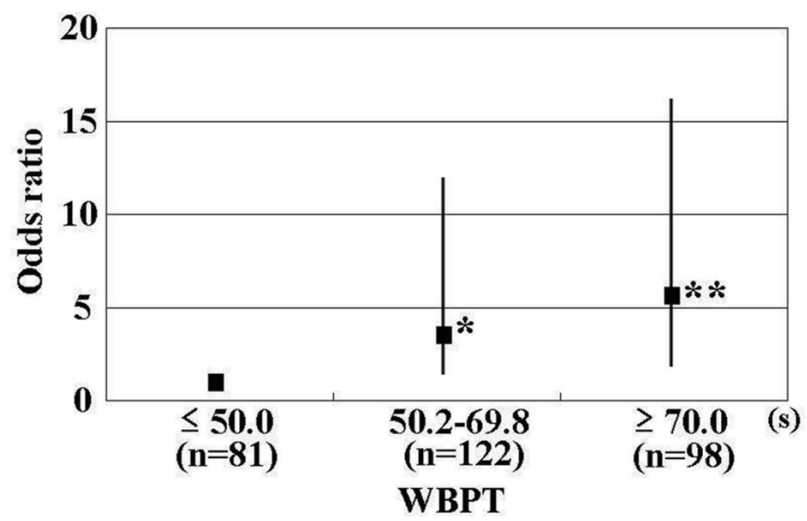

Figure 3. Results of multiple logistic regression analysis for high hscTnT. Patients with high ( $\geq 70 \mathrm{~s}$; odds ratio, $5.3(95 \% \mathrm{Cl}, 1.6-16.1)$; $\mathrm{P}$ $<0.01$ ) and median (50.2 - $69.8 \mathrm{~s}$; odds ratio, 3.8 (95\% Cl, 1.2 - 12.4); $\mathrm{P}$ $<0.05$ ) WBPT had a significantly higher risk for high hs-cTnT levels ( $\geq$ $0.014 \mathrm{ng} / \mathrm{mL}$ ) than those with low WBPT ( $\leq 50.0 \mathrm{~s})$. Adjustment factors: CAVI, log-BNP, skin AF, d-ROMs test, age. ${ }^{*} \mathrm{P}<0.05 \mathrm{vs}$. $\leq 50.0 \mathrm{~s}$, ${ }^{*} \mathrm{P}<$ 0.01 vs. $\leq 50.0$ s. Cl: confidence interval; CAVI: cardio-ankle vascular index; BNP: brain natriuretic peptide; AF: autofluorescence; d-ROMs: derivatives of reactive oxygen metabolites. 
patients without diabetes mellitus or healthy controls are approximately 40 s degree $[23,24]$, whereas mean WBPT values in this study were $62.5 \mathrm{~s}$. Thus, these results indicated that the impairment of hemorheology in patients with type 2 diabetes mellitus was progressive compared with that in nondiabetic or healthy controls. Furthermore, independent associations between WBPT and hs-cTnT levels suggest that the impairment of hemorheology is an important factor for the progression of subclinical myocardial injury or primary cardiovascular incidence in patients with type 2 diabetes mellitus. Skin AF, dROMs test, and CAVI were selected as independent variables for both hs-cTnT and WBPT as subordinate factors.

Among the glucose-related parameters, only skin AF was selected as an independent variable when hs-cTnT was a subordinate factor. In addition, skin AF also had an independent association with WBPT. Skin AF was reported to reflect pentosidine levels, which are major components of AGEs on the volar side of the lower arm by study of skin biopsy [25]. Hemorheology assessed using MC-FAN is an in vitro measurement that uses artificial blood vessels, with a $7 \mu \mathrm{m}$ wide, $30 \mu \mathrm{m}$ long, and $4.5 \mu \mathrm{m}$ deep vessel lumen. Thus, assessing hemorheology using MC-FAN is assumed to correspond to small vessels. Small vessels are considered to be present in a portion of skin biopsies along with systemic vessels, including heart vessels. Therefore, the impairment of hemorheology is a possible cause of the accumulation of AGEs in systemic tissues, including the heart. In addition, Hofmann et al have revealed a significant relationship between AGE-modified cardiac tissue collagen and skin AF [26]. Several pathways by which AGE or the receptor of AGE influences myocardial injury in diabetes models have been reported [27, 28]. Furthermore, basic studies have indicated that AGEs influence hemorheology by mechanisms such as leukocyte-endothelial interaction and platelet aggregation $[29,30]$. Thus, the results of this and previous studies may indicate that hemorheology and AGEs are associated not only on the volar side of the lower arm but also in small cardiac vessels, consequently leading to myocardial injury in patients with type 2 diabetes mellitus.

Oxidative stress is well known to be closely associated with the pathogenesis of diabetes mellitus. Our results also indicated that d-ROMs test, as a marker of oxidative stress, was an important factor for both myocardial injury and hemorheology in patients with type 2 diabetes mellitus with no history of cardiovascular events. Several pathways have been identified by which oxidative stress caused myocardial injury, such as the dysfunction of the mitochondrial electron transport complex, activity of nicotinamide adenine dinucleotide phosphate oxidase, and apoptosis of myocardial cells $[3,31]$. In addition, several mechanisms are also reported that oxidative stress causes the impairment of hemorheology such as platelet aggregation and elevation of plasma viscosity $[32,33]$. Thus, oxidative stress is an important target factor to prevent both the progression of myocardial injury and impairment of hemorheology in patients with type 2 diabetes mellitus.

CAVI is known to be a marker of arterial stiffness, which is independently associated with blood pressure levels [17]. In recent years, several studies have reported the significance of left ventricular dysfunction in the progression of myocardial injury. Furthermore, increases in aortic artery stiffness are known to be caused by left ventricular dysfunction [34]. Therefore, the independent relationship between CAVI and hs-cTnT identified in this study was thought to reflect myocardial injury via left ventricular dysfunction resulting from an increase in vascular resistance or afterload. In addition, Endo et al have reported that CAVI reflected endothelial function [35]. Endothelial dysfunction has also been known to cause left ventricular dysfunction or myocardial injury [36, 37]. Thus, results of this study indicated the importance of arterial dysfunction, such as increase of arterial stiffness, or endothelial dysfunction on subclinical myocardial injury in patients with type 2 diabetes mellitus. Conversely, CAVI was also selected as an independent variable for WBPT as a subordinate factor in multivariate analysis. Some studies have reported the relationships between WBPT and arterial function such as arterial stiffness, endothelial function, and vascular resistance [11, 24, 38, 39]. Therefore, increased WBPT due to increased hs-cTnT levels may be partly explained by arterial dysfunction.

It is useful to have simple cut-off levels of WBPT values for predicting abnormal hs-cTnT levels in clinical settings. This study clarifies the clinical usefulness of assessing WBPT to detect hs-cTnT levels as high as $\geq 0.014 \mathrm{ng} / \mathrm{mL}$, which is shown to be discriminatory of increased hs-cTnT levels [5]. Patients were divided into three groups based on simple WBPT values, and multiple logistic regression analysis was performed. Our results indicated that patients with WBPT of $\geq 70.0 \mathrm{~s}$ or $50.2-69.8 \mathrm{~s}$ exhibit a significantly higher risk of high hs-cTnT levels than those with WBPT $\leq 50.0 \mathrm{~s}$. Although this was a cross-sectional study, we believe that it is possible to evaluate the risk of the progression of high hs-cTnT levels in patients with type 2 diabetes mellitus by measuring WBPT. Some clinical studies have indicated that medications, such as antidiabetic, antihypertensive, and antihyperlipidemic drugs, and smoking cessation improve hemorheology [40-43]. In this cross-sectional study, smoking habits were independently associated with WBPT, although no significant association was observed between medication and WBPT. We suggest that an increase in hs-cTnT levels can be prevented by interventions, such as smoking cessation, or medication in patients with type 2 diabetes mellitus to maintain WBPT at $\leq 50.0 \mathrm{~s}$.

This study has several limitations. First, drug treatments for diabetes mellitus, hypertension, and dyslipidemia may have influenced the results. Second, HOMA-IR has limitations as a marker of insulin resistance, particularly in patients with high blood glucose levels. This study included a substantial number of patients with type 2 diabetes mellitus with high fasting blood glucose levels. Therefore, additional studies using some other accurate insulin resistance marker, such as a glucose clamp test, are warranted to evaluate the significance of insulin resistance on hemorheology or hs-cTnT in patients with type 2 diabetes mellitus. Third, angiography, computed tomography, magnetic resonance imaging, and echocardiography were not performed; thus, asymptomatic cardiovascular diseases may have remained undetected. Forth, hemorheology assessed using MC-FAN is an in vitro assessment that uses artificial blood vessels; therefore, hemorheological data obtained might differ from those obtained in vivo because of the influence of vascular factors, such as endothelial or smooth 
muscle cells. However, the results of this study indicate that WBPT is an important marker associated with an increase in hs-cTnT levels or a risk factor for the progression of subclinical myocardial injury or cardiovascular disease incidence in patients with type 2 diabetes mellitus. Finally, this study was cross-sectional and conducted at a single facility with a small sample size. Future prospective studies, including intervention therapies, are required to confirm our results.

\section{Conclusions}

The results of this study indicate the independent associations of hemorheology with hs-cTnT in patients with type 2 diabetes mellitus assessed by the MC-FAN. We suggest that an increase in hs-cTnT levels can be prevented by maintaining WBPT at $\leq$ 50.0 s. Future additional prospective studies, including intervention therapies, are required to confirm our results.

\section{Competing Interests}

Author has no competing interests.

\section{Grant Support}

None.

\section{References}

1. Selvin E, Lazo M, Chen Y, Shen L, Rubin J, McEvoy JW, Hoogeveen RC, et al. Diabetes mellitus, prediabetes, and incidence of subclinical myocardial damage. Circulation. 2014;130(16):1374-1382.

2. Pitkanen OP, Nuutila P, Raitakari OT, Ronnemaa T, Koskinen PJ, Iida H, Lehtimaki TJ, et al. Coronary flow reserve is reduced in young men with IDDM. Diabetes. 1998;47(2):248-254.

3. Li SY, Yang X, Ceylan-Isik AF, Du M, Sreejayan N, Ren J. Cardiac contractile dysfunction in Lep/Lep obesity is accompanied by NADPH oxidase activation, oxidative modification of sarco(endo)plasmic reticulum $\mathrm{Ca} 2+-\mathrm{AT}$ Pase and myosin heavy chain isozyme switch. Diabetologia. 2006;49(6):1434-1446.

4. Twerenbold R, Jaffe A, Reichlin T, Reiter M, Mueller C. High-sensitive troponin $\mathrm{T}$ measurements: what do we gain and what are the challenges? Eur Heart J. 2012;33(5):579-586.

5. Saunders JT, Nambi V, de Lemos JA, Chambless LE, Virani SS, Boerwinkle E, Hoogeveen RC, et al. Cardiac troponin $\mathrm{T}$ measured by a highly sensitive assay predicts coronary heart disease, heart failure, and mortality in the Atherosclerosis Risk in Communities Study. Circulation. 2011;123(13):1367-1376.

6. Otsuka T, Kawada T, Ibuki C, Seino Y. Association between high-sensitivity cardiac troponin $\mathrm{T}$ levels and the predicted cardiovascular risk in middle-aged men without overt cardiovascular disease. Am Heart J. 2010;159(6):972-978.

7. Resl M, Clodi M, Vila G, Luger A, Neuhold S, Wurm R, Adlbrecht C, et al. Targeted multiple biomarker approach in predicting cardiovascular events in patients with diabetes. Heart. 2016;102(24):1963-1968.

8. Tzoulaki I, Murray GD, Lee AJ, Rumley A, Lowe GD, Fowkes FG. Relative value of inflammatory, hemostatic, and rheological factors for incident myocardial infarction and stroke: the Edinburgh Artery Study. Circulation. 2007;115(16):2119-2127.

9. Cowan AQ, Cho DJ, Rosenson RS. Importance of blood rheology in the pathophysiology of atherothrombosis. Cardiovasc Drugs Ther. 2012;26(4):339-348.

10. Kikuchi Y, Sato K, Mizuguchi Y. Modified cell-flow microchannels in a single-crystal silicon substrate and flow behavior of blood cells. Microvasc Res. 1994;47(1):126139.

11. Satoh N, Kotani K, Wada H, Himeno A, Shimada S, Sasaki Y, Yamada K, et al. Unfavorable blood rheology is closely associated with arterial stiffness in obese patients. Endocr J. 2009;56(7):915-918.

12. Hitsumoto T. Factors affecting impairment of blood rheology in obese subjects. J Cardiol. 2012;60(5):401-406.

13. Matsuo K, Ueda Y, Nishio M, Hirata A, Asai M, Nemoto T, Kashiwase K, et al. Thrombogenic potential of whole blood is higher in patients with acute coronary syndrome than in patients with stable coronary diseases. Thromb Res. 2011;128(3):268-273.

14. Hitsumoto T. Relationship between cardiovascular risk factors and hemorheology assessed by microchannel method in patients with type 2 diabetes mellitus. Diabetology International. 2017;8(3):316-322.

15. Meerwaldt R, Links TP, Graaff R, Hoogenberg K, Lefrandt JD, Baynes JW, Gans RO, et al. Increased accumulation of skin advanced glycation end-products precedes and correlates with clinical manifestation of diabetic neuropathy. Diabetologia. 2005;48(8):1637-1644.

16. Okin PM, Devereux RB, Liu JE, Oikarinen L, Jern S, Kjeldsen SE, Julius S, et al. Regression of electrocardiographic left ventricular hypertrophy predicts regression of echocardiographic left ventricular mass: the LIFE study. J Hum Hypertens. 2004;18(6):403-409.

17. Shirai K, Utino J, Otsuka K, Takata M. A novel blood pressure-independent arterial wall stiffness parameter; cardio-ankle vascular index (CAVI). J Atheroscler Thromb. 2006;13(2):101-107.

18. Friedewald WT, Levy RI, Fredrickson DS. Estimation of the concentration of low-density lipoprotein cholesterol in plasma, without use of the preparative ultracentrifuge. Clin Chem. 1972;18(6):499-502.

19. Matthews DR, Hosker JP, Rudenski AS, Naylor BA, Treacher DF, Turner RC. Homeostasis model assessment: insulin resistance and beta-cell function from fasting plasma glucose and insulin concentrations in man. Diabetologia. 1985;28(7):412-419.

20. Imai E, Horio M, Nitta K, Yamagata K, Iseki K, Hara $\mathrm{S}$, Ura $\mathrm{N}$, et al. Estimation of glomerular filtration rate 
by the MDRD study equation modified for Japanese patients with chronic kidney disease. Clin Exp Nephrol. 2007;11(1):41-50.

21. Cesarone MR, Belcaro G, Carratelli M, Cornelli U, De Sanctis MT, Incandela L, Barsotti A, et al. A simple test to monitor oxidative stress. Int Angiol. 1999;18(2):127-130.

22. Mingels A, Jacobs L, Michielsen E, Swaanenburg J, Wodzig W, van Dieijen-Visser M. Reference population and marathon runner sera assessed by highly sensitive cardiac troponin $\mathrm{T}$ and commercial cardiac troponin $\mathrm{T}$ and I assays. Clin Chem. 2009;55(1):101-108.

23. Yoshida K, Kimura T, Aoki T, Tsunekawa K, Araki O, Shoho Y, Nara M, et al. Fasting serum insulin levels and insulin resistance are associated with blood rheology in Japanese young adults without diabetes. J Int Med Res. 2016;44(3):496-507.

24. Yagi H, Sumino H, Aoki T, Tsunekawa K, Araki O, Kimura T, Nara M, et al. Impaired blood rheology is associated with endothelial dysfunction in patients with coronary risk factors. Clin Hemorheol Microcirc. 2016;62(2):139150 .

25. Meerwaldt R, Graaff R, Oomen PHN, Links TP, Jager JJ, Alderson NL, Thorpe SR, et al. Simple non-invasive assessment of advanced glycation endproduct accumulation. Diabetologia. 2004;47(7):1324-1330.

26. Hofmann B, Jacobs K, Navarrete Santos A, Wienke A, Silber RE, Simm A. Relationship between cardiac tissue glycation and skin autofluorescence in patients with coronary artery disease. Diabetes Metab. 2015;41(5):410-415.

27. Ma H, Li SY, Xu P, Babcock SA, Dolence EK, Brownlee M, Li J, et al. Advanced glycation endproduct (AGE) accumulation and AGE receptor (RAGE) up-regulation contribute to the onset of diabetic cardiomyopathy. J Cell Mol Med. 2009;13(8B):1751-1764.

28. Brett J, Schmidt AM, Yan SD, Zou YS, Weidman E, Pinsky D, Nowygrod R, et al. Survey of the distribution of a newly characterized receptor for advanced glycation end products in tissues. Am J Pathol. 1993;143(6):1699-1712.

29. Morigi M, Angioletti S, Imberti B, Donadelli R, Micheletti G, Figliuzzi M, Remuzzi A, et al. Leukocyte-endothelial interaction is augmented by high glucose concentrations and hyperglycemia in a NF-kB-dependent fashion. J Clin Invest. 1998;101(9):1905-1915.

30. Hasegawa Y, Suehiro A, Higasa S, Namba M, Kakishita E. Enhancing effect of advanced glycation end products on serotonin-induced platelet aggregation in patients with diabetes mellitus. Thromb Res. 2002;107(6):319-323.

31. Boudina S, Abel ED. Diabetic cardiomyopathy, causes and effects. Rev Endocr Metab Disord. 2010;11(1):31-39.
32. Tzoulaki I, Murray GD, Lee AJ, Rumley A, Lowe GD, Fowkes FG. Inflammatory, haemostatic, and rheological markers for incident peripheral arterial disease: Edinburgh Artery Study. Eur Heart J. 2007;28(3):354-362.

33. Podrez EA, Byzova TV, Febbraio M, Salomon RG, Ma Y, Valiyaveettil M, Poliakov E, et al. Platelet CD36 links hyperlipidemia, oxidant stress and a prothrombotic phenotype. Nat Med. 2007;13(9):1086-1095.

34. Jaroch J, Loboz Grudzien K, Bociaga Z, Kowalska A, Kruszynska E, Wilczynska M, Dudek K. The relationship of carotid arterial stiffness to left ventricular diastolic dysfunction in untreated hypertension. Kardiol Pol. 2012;70(3):223-231.

35. Endo K, Saiki A, Ohira M, Miyashita Y, Shirai K. Cardioankle vascular index may reflect endothelial function in type 2 diabetes. Int J Clin Pract. 2011;65(11):1200-1201.

36. Zizek B, Poredos P. Increased left ventricular mass and diastolic dysfunction are associated with endothelial dysfunction in normotensive offspring of subjects with essential hypertension. Blood Press. 2007;16(1):36-44.

37. Marti V, Aymat R, Ballester M, Garcia J, Carrio I, Auge JM. Coronary endothelial dysfunction and myocardial cell damage in chronic stable idiopathic dilated cardiomyopathy. Int J Cardiol. 2002;82(3):237-245.

38. Hitsumoto $T$. Impact of hemorheology assessed by the microchannel method on pulsatility index of the common carotid artery in patients with type 2 diabetes mellitus. J Clin Med Res. 2017;9(7):579-585.

39. Hitsumoto T. Relationship between hemorheology assessed using microchannel array flow analyzer and kidney function in hypertensive patients. Cardiol Res. 2017;8(4):147-153.

40. Ott C, Raff U, Schmidt S, Kistner I, Friedrich S, Bramlage $\mathrm{P}$, Harazny JM, et al. Effects of saxagliptin on early microvascular changes in patients with type 2 diabetes. Cardiovasc Diabetol. 2014;13:19.

41. Yamada K, Hirayama T, Hasegawa Y. Antiplatelet effect of losartan and telmisartan in patients with ischemic stroke. J Stroke Cerebrovasc Dis. 2007;16(5):225-231.

42. Tirnaksiz E, Pamukcu B, Oflaz H, Nisanci Y. Effect of high dose statin therapy on platelet function; statins reduce aspirin-resistant platelet aggregation in patients with coronary heart disease. J Thromb Thrombolysis. 2009;27(1):24-28.

43. Shimada S, Hasegawa K, Wada H, Terashima S, SatohAsahara N, Yamakage H, Kitaoka S, et al. High blood viscosity is closely associated with cigarette smoking and markedly reduced by smoking cessation. Circ J. 2011;75(1):185-189. 